\title{
Photoemission Microscopy for Integrated Photonics
}

\author{
R. C. Word, J. P. S. Fitzgerald, and R. Könenkamp
}

Department of Physics, Portland State University, Portland, OR, USA

Parallel developments in ultrafast lasers, high sensitivity CCD cameras, and aberration correction have led to the development of practical nonlinear multi-photon photoelectron emission microscopy (PEEM). In multi-photon photoelectron emission small differences in the local field, which can be very large in the fs regime, can generate high photoelectron contrast. Contrast, brightness, and resolution are further enhanced by corrected optics tailored to the high chromatic aberration of PEEM, which was pioneered with the mirror-based design of Gertrude Rempfer and coworkers [1]. The most fruitful application of multiphoton PEEM is presently the study of surface plasmon polaritons (SPP). These are surface electromagnetic waves that can occur at the interface between metals and dielectrics and have many possible applications. In PEEM, we image electrons generated by interference between a SPP and the generating incident laser light (or secondary probe beam). Published reports include images of SPP waves confined to nanowires [2] and antennae milled from nanoflakes [3, 4], as well as the distribution of localized SPP 'hotspots' on diffraction gratings [5] and randomly patterned gold films [6].

In addition to plasmonic phenomena, photonic phenomena such as diffraction from particles or edges are familiar image features in PEEM. These so-called 'fringe fields' can be used to obtain information on the diffracting object [7], but are usually ignored as image artifacts. Recently we found that in addition to diffraction, and similar to near field scanning optical microscopy [8], PEEM can be used to image the near field of a photonic wave bound to indium tin oxide (ITO) thin film. The nontrivial absorption of light in the waveguide can be compensated for by local coupling of light through a surface defect such as a slot, particle, or hole. The damped guided photonic wave can be observed in PEEM traveling for tens of microns. With a reasonably long propagation length there is the possibility of using PEEM to study a photonic wave in a photonic circuit. Perhaps most interesting is the possibility of observing a guided photonic wave that, through dielectric $k$-matching, couples into the metal as a SPP, which we might also see in PEEM (Fig 1a).

Here we report the use of PEEM for the visualization of photonic guided waves, coupling of photonic waves into SPPs, propagating SPPs on gold created by edge diffraction, and localized SPPs bound to gold quantum dots and nanorods in a single image frame. The presence of many types of optical phenomena makes description and analysis complicated. Briefly, ITO is opaque in the UV and does not support a bound photonic wave (Fig 1b). We see ordinary topographical and work function contrast, as well as diffraction and SPPs generated in the near field of diffraction at the edges of many flakes (particularly flake F1). Similar SPP wave patterns appear in 2-photon photoemission PEEM (Fig 1c and 1d). Depending on the angle of the flakes' edges, SPPs can be generated in the near field of diffraction in both $\mathrm{S}$ and $\mathrm{P}$ polarizations.

At $\lambda=410 \mathrm{~nm}$ ITO is transparent and bound photonic waves are a major image feature. When the incident light is P-polarized (Fig 1c) we see that the small gold flake (F2) can support a SPP coupled into it by dielectric $k$-matching from the TM mode below. The photonic wave suffers high losses due to SPP coupling to the gold. When the light is S-polarized (Fig 1d), we see that the guided TE wave cannot couple into the smaller flake (F2) as a SPP. The flake is dark. We also note that while the overall 
emission rate is low, localized surface plasmon resonance makes the quantum dot and nanorod exceptionally bright.

Direct imaging of photonic and plasmonic waves and the coupling between them is an important demonstration of the use of PEEM in the study of integrated photonic circuits. In ongoing work we hope to tailor the photonic waveguide and metal structures to produce more intentional outcomes.

Experimental details:

Gold nanoflakes were grown using the auric acid method of [9] and drop deposited onto a glass coverslip coated with a 240-nm thick layer of ITO with surface resistivity of $15 \Omega / \square$. Near a selection of gold flakes we milled, by FIB, a 300-nm deep trench as our waveguide input interface. The specimen was imaged in our home-built $20-\mathrm{kV}$ aberration-corrected PEEM. Illumination was by a 244-nm argon ion laser for UV PEEM or a frequency doubled 410-nm Ti:sapphire laser with incident power $150 \mathrm{~mW}$ and pulse length 80-100 fs for two-photon PEEM. Laser polarization was set using a tunable waveplate. Exposure times are $30 \mathrm{sec}$. for UV and $5 \mathrm{~min}$. for blue.

\section{References:}

[1] R. Könenkamp et al. Ultramicroscopy, 110 (2010) p. 899.

[2] L Douillard et al. Nano Lett. 8 (2008) p. 935.

[3] JS Huang et al. Nat. Commun. 1 (2010) p. 150.

[4] RC Word, JPS Fitzgerald, and R Könenkamp, Surf. Sci. 607 (2013) p. 148.

[5] A Kubo et al. J. Phys. B: At., Mol. Opt. Phys. 40 (2007) p. S259.

[6] RC Word, J Fitzgerald, and R Könenkamp, Appl. Phys. Lett. 99 (2011) 041106.

[7] LI Chelaru et al. Phys. Rev. B 73 (2006) p. 115416.

[8] E Flück et al. J. of Lightwave Technol. 21 (2003) p. 1384.

[9] Z Guo et al. Colloids Surf. A 278 (2006) p. 33.

[10] This work was supported by the US-DOE Basic Science Office contract DE-FG02-10ER46406.

(a)

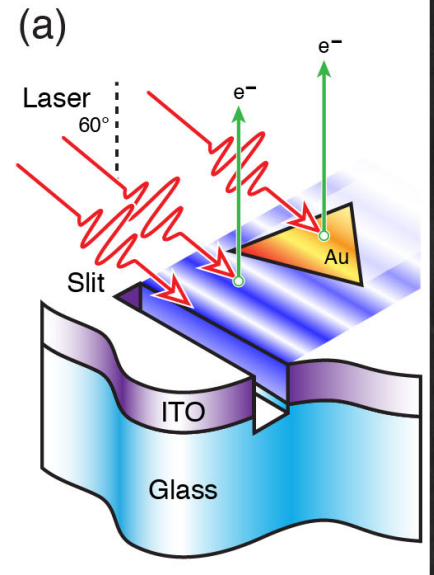

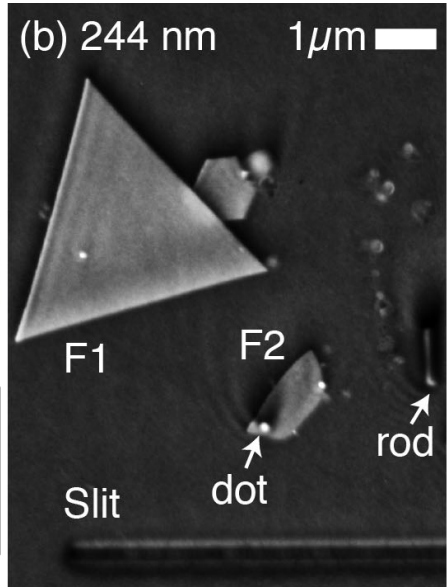

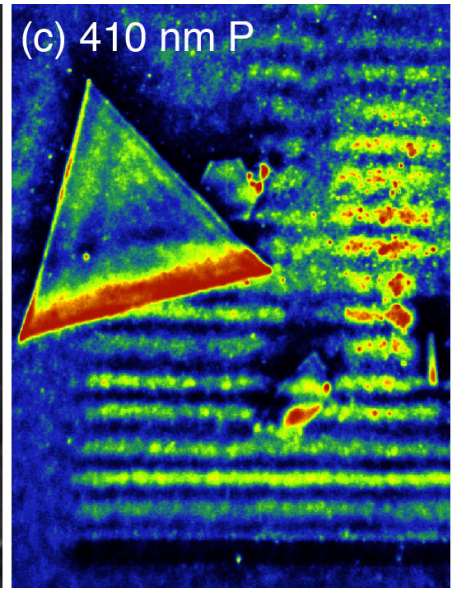

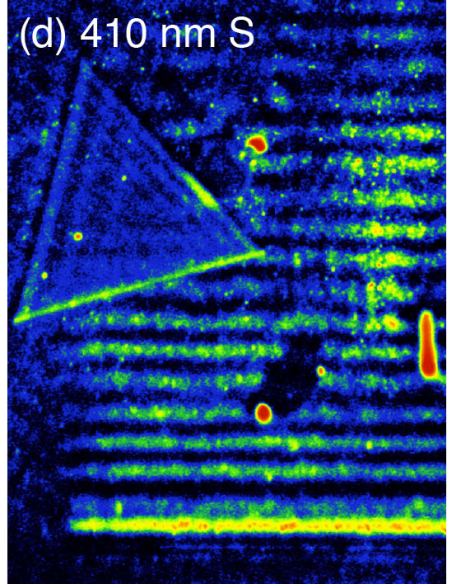

Figure 1. (a) Imaging photoelectrons are emitted as the incident laser interferes with guided photonic waves coupled into a slit or plasmonic waves bound to gold. (b) UV PEEM of gold flakes on ITO. (c) False color two-photon PEEM taken with 410-nm P-polarized light and (d) S-polarized light. 